\title{
Morbidade materna grave na microrregião de Barbacena/MG
}

\author{
Severe maternal morbidity in the microregion of \\ Barbacena, Minas Gerais state, Brazil
}

\author{
Carlos Eduardo Leal Vidal' ${ }^{1}$, Marco Aurélio Bernardes de Carvalho', \\ Izabela Ribeiro Grimaldi ${ }^{1}$, Marcela Campos dos Reis ${ }^{1}$, \\ Maria Clara Nepomuceno Baêta ${ }^{1}$, Raíssa Barbosa Garcia ${ }^{1}$, \\ Senice Alvarenga Rodrigues Silva ${ }^{1}$
}

\begin{abstract}
Resumo
Introdução: As complicações da gestação, parto e puerpério somam a décima causa de morte feminina e constituem um problema de saúde pública nos países menos desenvolvidos. Objetivo: O objetivo deste estudo foi o de identificar os fatores associados à morbidade materna grave na microrregião de Barbacena, em Minas Gerais, no período de fevereiro a agosto de 2014. Métodos: Estudo tipo caso-controle, no qual foram selecionadas como casos todas as puérperas que preencheram pelo menos um dos critérios para a morbidade e como controles as que não apresentaram nenhuma intercorrência. $A$ análise dos fatores associados à morbidade foi realizada por meio de modelo de regressão logística. O nível de significância adotado foi de 5\%. Resultados: Foram avaliadas 276 mulheres, das quais 92 eram casos, e 184, controles. Na análise multivariada do modelo final de regressão logística, permaneceram as variáveis: história pregressa de hipertensão arterial $(O R=20,8 ;$ IC95\%=6,4-67,4), prematuridade $(\mathrm{OR}=7,5$; IC95\%=3,8-14,8) e parto cesáreo $(\mathrm{OR}=3,4$; IC95\%=1,69-6,76). Conclusão: Houve uma relação forte entre as variáveis pesquisadas no presente estudo e a ocorrência de eventos adversos, com a importância da identificação precoce dos fatores de risco para morbidade materna e o estabelecimento de estratégias preventivas.
\end{abstract}

Palavras-chave: morbidade; complicações na gravidez; puerpério.

\begin{abstract}
Background: Complications during pregnancy, birth, and puerperium add up to the tenth death cause among women, and constitute a public health problem in developing countries. Objective: The objective of this study was to identify the factors associated with severe maternal morbidity in the microregion of Barbacena, Minas Gerais state, Brazil from February to August 2014. Methods: This is a case-control study where all puerperal women who met at least one criterion of morbidity were selected as case, whereas those who did not present any complications were considered as control. The factors associated with morbidity were analyzed using the logistic regression model at 5\% significance level. Results: The study assessed 276 women, of which 92 were cases and 184 were controls. In the multivariate analysis of the final logistic regression model, the variables that persisted were past medical history of high blood pressure ( $\mathrm{OR}=20.8$; $\mathrm{Cl} 95 \%$ : 6.4-67.4), prematurity (OR=7.5; $\mathrm{Cl} 95 \%$ : 3.8-14.8), and caesarean section (OR=3.4; Cl 95\%: 1.69-6.76).

Conclusions: A strong correlation was found between the variables investigated and the occurrence of adverse events, accentuating the importance of early identification of risk factors for maternal morbidity and establishment of preventive strategies.

Keywords: morbidity; pregnancy complications; puerperium.
\end{abstract}

${ }^{1}$ Faculdade de Medicina de Barbacena (FAME) - Barbacena (MG), Brasil.

${ }^{2}$ Serviço de Gestação de Alto Risco da Santa Casa de Barbacena - Barbacena (MG), Brasil.

Trabalho realizado na Santa Casa de Misericórdia de Barbacena - Barbacena (MG), Brasil.

Endereço para correspondência: Carlos Eduardo Leal Vidal - Rua Professor Vasconcelos, 467 - CEP: 36205-238 - Barbacena (MG), Brasil - Email: celv@uol.com.br

Fonte de financiamento: nenhuma.

Conflito de interesses: nada a declarar. 


\section{INTRODUÇÃO}

A mortalidade materna é um importante indicador de desenvolvimento humano/social e merece destaque por comparar níveis de desenvolvimento entre países ou regiões, revelando mais claramente as desigualdades sociais. Esse marcador abrange qualquer causa de morte relacionada ou agravada pela gravidez e exclui óbitos por causas acidentais ou incidentais ${ }^{1-4}$. O óbito materno é mais frequente em mulheres de classe social desfavorecida em termos de renda, escolaridade, acesso a serviços de saúde de qualidade e à informação sobre planejamento familiar ${ }^{4}$.

De acordo com a Organização Mundial da Saúde (OMS), em 20 anos houve uma redução significativa no número de mortes maternas, caindo de 543 mil mortes em 1990 para 287 mil em $2010^{5}$. Mesmo que significativo, esse decréscimo corresponde a menos da metade do estipulado para o cumprimento da meta de redução, que era de 75\% entre 1990 e 2015, de acordo com os Objetivos de Desenvolvimento do Milênio ${ }^{5}$.

Apesar de a mortalidade materna estar apresentando declínio contínuo nos países desenvolvidos, ainda é um problema importante e aparentemente de difícil resolução nos países subdesenvolvidos ou em desenvolvimento ${ }^{4,6}$. Como exemplo, a razão de mortalidade materna (RMM) é estimada em 6 óbitos para cada 100 mil nascidos vivos (NV) no Canadá, enquanto que no Haiti esse valor corresponde a 680 óbitos $/ 100 \mathrm{mil} \mathrm{NV}^{2}$.

Em 20 anos, o Brasil reduziu a RMM em 53\%, passando de 120 óbitos/100 mil NV em 1990 para 56 óbitos/100 mil NV em 2010. A OMS recomenda que a RMM aceitável esteja entre 10 e 20 óbitos/100 mil NV ${ }^{2,7}$. Em Barbacena, no Estado de Minas Gerais, entre 1998 e 2012 houve uma redução de 59\% da RMM, passando de 132 para 54 óbitos/100 mil NV 8

Além da morte, um considerável número de mulheres desenvolve morbidade grave durante a gestação, parto ou puerpério. Os agravos ocorridos nesse período compreendem, entre outros, síndromes hipertensivas, infecções, hemorragia e problemas cardiovasculares. A morbidade resultante de complicação grave em obstetrícia é referida pela expressão inglesa "near miss", cuja tradução literal é "quase perda". O conceito de near miss refere-se então a morbidades graves, potencialmente letais, porém que não resultaram em morte en, $3,6,9-11^{\text {. }}$.

As morbidades associadas ao conceito do near miss são de fundamental importância para o estudo da morbimortalidade materna, pois representam um grupo muito mais prevalente que os casos de morte materna, principalmente em países desenvolvidos ${ }^{10-12}$. O estudo desses agravos é importante porque auxilia no entendimento sobre a assistência obstétrica prestada em diferentes contextos, além de propiciar possíveis soluções para minimizar o problema ${ }^{6}$.

Com base nesses dados e na relevância do tema, o objetivo deste estudo foi identificar os fatores associados à morbidade

materna grave na microrregião de Barbacena, em Minas Gerais, no período de fevereiro a agosto de 2014.

\section{MÉTODOS}

\section{Tipo e local do estudo}

Foi realizado um estudo tipo caso-controle, de base hospitalar, na proporção de dois controles para cada caso, na microrregião de Barbacena, cuja composição é de 15 municípios e compreende uma população aproximada de 230 mil habitantes. Foram avaliadas todas as puérperas que preencheram os critérios de morbidade internadas na maternidade da Santa Casa de Misericórdia de Barbacena. O referido hospital é responsável por cerca de $2 \mathrm{mil}$ nascimentos anuais e é referência regional para o atendimento de alto risco obstétrico.

\section{Amostra}

Todas as pacientes internadas e que tiveram complicações durante a gestação ou puerpério e que cumpriram pelo menos um dos requisitos propostos pelos critérios da OMS foram consideradas como casos de morbidade materna grave $e^{6,9,10,12-14}$. Esses critérios são apresentados na Tabela 1. O grupo controle foi constituído por puérperas que não preencheram os critérios de diagnósticos estabelecidos, que estavam internadas no mesmo hospital dos casos e que foram pareadas por plantão. Foram excluídas mulheres internadas por abortamento. O tamanho da amostra foi calculado como 92 casos e 184 controles, tendo

Tabela 1. Condições potencialmente ameaçadoras à vida da mulher no ciclo grávido-puerperal ${ }^{6}$

Distúrbios hemorrágicos

Descolamento prematuro de placenta

Placenta acreta, increta ou percreta

Prenhez ectópica

Hemorragia pós-parto

Rotura uterina

Outros distúrbios sistêmicos

Endometrite

Edema pulmonar

Insuficiência respiratória

Convulsões

Sepse

Choque

Trombocitopenia $<100$ mil

Crise tireotóxica

Fonte: Dias et al. ${ }^{6}$

\section{Distúrbios hipertensivos}

Pré-eclâmpsia grave

Eclâmpsia

Hipertensão grave

Encefalopatia hipertensiva

Síndrome HELLP

Indicadores de gravidade de manejo

Transfusão sanguínea

Acesso venoso central

Histerectomia

Admissão à unidade de tratamento intensivo Internação hospitalar prolongada ( $>7$ dias pós-parto) Intubação não anestésica

Retorno à sala operatória (centro cirúrgico) Intervenção cirúrgica 
por base um Odds Ratio esperado igual ou maior que 2,5, poder de $80 \%$, nível de significância de 5\% e prevalência esperada de baixa cobertura pré-natal entre os controles de $15 \%{ }^{15}$. Pelo fato de a morbidade materna grave ser uma ocorrência pouco frequente, escolheu-se o pareamento 1:2 para casos e controles com o objetivo de elevar o poder do estudo ${ }^{16}$.

\section{Procedimentos}

Foi elaborado um questionário para a coleta de dados, no qual, inicialmente, foram apuradas condições potencialmente ameaçadoras à vida da puérpera, a fim de identificar os casos, conforme definição da OMS (Tabela 1). Para isso, foram analisadas, primeiramente, pacientes que apresentassem um ou mais dos seguintes fatores: a ocorrência de distúrbios hipertensivos, hemorrágicos, sistêmicos, disfunção cardiovascular, respiratória, renal, hematológica e hemostática, hepática e neurológica. Os indicadores de gravidade de manejo, como transfusão sanguínea, acesso venoso central, histerectomia, admissão à UTI, internação hospitalar prolongada (maior que sete dias pós-parto), intubação não anestésica, retorno à sala operatória e intervenção cirúrgica, também foram abordados na entrevista e priorizados na análise de dados. Com o objetivo de correlacionar outras possíveis condições desencadeadoras de comorbidades, foram coletados secundariamente dados sociodemográficos (idade, estado civil, procedência, profissão, escolaridade, renda) e clínicos (etilismo, tabagismo, uso de drogas, morbidades clínicas, uso de medicação, vacinação, história de hipertensão e diabetes prévios. Para o álcool foi perguntado sobre a frequência de uso, considerando resposta positiva quando mencionado o uso de uma ou mais doses de bebida pelo menos uma vez por semana. Em relação ao tabagismo, foi perguntado apenas se fumava ou não e se o uso era diário, independente da quantidade consumida. Quanto aos uso de drogas ilícitas, foi perguntado se eram consumidoras e se consumiram durante a gestação, sem especificar a quantidade. Também foi avaliada a história obstétrica das entrevistadas, com informações sobre gestação e parto pregressos, abortos prévios, número de consultas de pré-natal, tipo de parto, tempo de gestação, natimorto, complicações no parto e gestação gemelar. As puérperas responderam ao questionário imediatamente quando foram admitidas na enfermaria do hospital até $24 \mathrm{~h}$ após o parto. No caso das puérperas com morbidade grave, os questionários foram aplicados após resolução do quadro das respectivas e daquelas egressas da UTI. Foram incluídos os óbitos maternos ocorridos durante ou até 42 dias após o parto, por meio do cruzamento com o banco de dados do serviço de vigilância epidemiológica regional, que monitora os óbitos maternos.

\section{Análise estatística}

Os dados de cada participante foram registrados em fichas elaboradas especialmente para o estudo, posteriormente digitadas em planilhas do programa Excel, e processados por meio do software SPSS-17.0. Foi realizada estatística descritiva para caracterização da amostra segundo variáveis de interesse do estudo e foram construídas tabelas para distribuição de frequências, médias, medianas e desvio-padrão das variáveis. Foram utilizados testes de associações como o teste do qui-quadrado e o teste de Fischer para variáveis categóricas, além do teste $t$ de Student para variáveis contínuas. A análise dos fatores associados à morbimortalidade foi realizada por meio de modelo de regressão logística. Todas as variáveis que foram significativas no valor de 0,25 foram consideradas candidatas ao modelo multivariado ${ }^{17}$. Inicialmente, foi ajustado um modelo com todas essas variáveis candidatas, e, passo a passo, foram retiradas aquelas menos significativas, até ficarem no modelo somente as variáveis significativas com valor de 0,05 . A adequacidade do modelo foi avaliada pelo teste de Hosmer e Lemeshow. O nível de significância adotado foi de 5\%.

\section{Considerações éticas}

As mulheres foram entrevistadas depois da assinatura do termo de consentimento livre e esclarecido. As menores de idade assinaram um termo de assentimento com o responsável. O presente estudo foi aprovado pelo Comitê de Ética em Pesquisa da Fundação Hospitalar do Estado de Minas Gerais (FHEMIG) sob o n $\mathrm{n}^{\circ} 453.541$.

\section{RESULTADOS}

Foram avaliadas 276 mulheres, das quais 92 eram casos (33,3\%), e 184, controles (66,6\%). A idade variou entre 14 e 43 anos, com média igual a $28 \pm 3,2$ anos. A maioria das mulheres era casada (63,8\%), com escolaridade igual ou maior do que oito anos de estudo (54,3\%), renda de um a três salários mínimos $(49,6 \%)$ e provenientes da zona urbana $(79,3 \%)$, das quais $51,4 \%$ eram de Barbacena. Quanto à ocupação, 27,9\% estavam desempregadas e 7,6\% eram estudantes. Em relação ao uso de bebida alcoólica, 25,7\% afirmaram que a consumiam, das quais $6,9 \%$ ingeriram-na durante a gestação. Foi observado que $14,5 \%$ eram tabagistas e $10,1 \%$ fumaram no período gestacional. Não houve diferença com relação à frequência do uso entre os dois grupos de mulheres $(\mathrm{p}=0,68)$. Já para o uso de outras drogas, como crack e maconha, constatou-se que $1,4 \%$ das mulheres era consumidora e apenas $1,1 \%$ fez uso na gravidez. Esses dados são apresentados na Tabela 2.

Em relação à história clínica das entrevistadas, 8,7\% eram portadoras de hipertensão arterial e 1,8\% era diabética. Já para a história obstétrica, 269 mulheres $(97,5 \%)$ fizeram pré-natal e $79,7 \%$ tiveram mais de seis consultas. O parto cesáreo $(64,1 \%)$ 
Tabela 2. Distribuição de casos e controles de morbimortalidade materna de acordo com o perfil sociodemográfico, em Barbacena, Minas Gerais, 2014

\begin{tabular}{|c|c|c|c|c|}
\hline Variáveis & Casos & Controles & Valor $\mathbf{p}^{*}$ & OR (95\%IC) \\
\hline & n (\%) & n (\%) & & \\
\hline \multicolumn{5}{|l|}{ Idade } \\
\hline$<20$ & $13(32,5)$ & $27(67,5)$ & 0,96 & $1,02(0,47-2,15)$ \\
\hline $20-29$ & $47(32,0)$ & $100(68,0)$ & & $0,83(0,48-1,46)$ \\
\hline$\geq 30$ & $32(36,0)$ & $57(64,0)$ & & - \\
\hline \multicolumn{5}{|l|}{ Estado civil } \\
\hline Casada & $58(33,0)$ & $118(67,0)$ & 0,85 & $0,95(0,56-1,61)$ \\
\hline Solteira & $34(34,0)$ & $66(66,0)$ & & \\
\hline \multicolumn{5}{|l|}{ Procedência } \\
\hline Zona rural & $21(36,8)$ & $36(63,8)$ & 0,52 & $1,21(0,65-2,22)$ \\
\hline Zona urbana & $71(32,4)$ & $148(67,6)$ & & \\
\hline \multicolumn{5}{|l|}{ Profissão } \\
\hline Empregada & $59(33,1)$ & $119(66,9)$ & 0,99 & $0,97(0,55-1,72)$ \\
\hline Desempregada & $26(33,8)$ & $51(66,2)$ & & $1,01(0,36-2,99)$ \\
\hline Estudante & $7(33,3)$ & $14(66,7)$ & & - \\
\hline \multicolumn{5}{|l|}{ Escolaridade } \\
\hline$<8$ anos & $45(35,7)$ & $81(64,3)$ & 0,44 & $1,21(0,73-2,01)$ \\
\hline$\geq 8$ anos & $47(31,3)$ & $103(68,7)$ & & \\
\hline \multicolumn{5}{|l|}{ Renda } \\
\hline$<1$ & $45(34,6)$ & $85(65,4)$ & 0,73 & $1,08(0,65-1,79)$ \\
\hline $1-3$ & $45(32,8)$ & $92(67,2)$ & & $1,71(0,34-8,57)$ \\
\hline$>3$ & $2(22.2)$ & $7(77.8)$ & & - \\
\hline \multicolumn{5}{|l|}{ Bebida alcoólica } \\
\hline Sim & $26(36,6)$ & $45(63,4)$ & 0,49 & $1,21(0,69-2,14)$ \\
\hline Não & $66(32,2)$ & $139(67,8)$ & & \\
\hline \multicolumn{5}{|l|}{ Tabagismo } \\
\hline Sim & $12(30.0)$ & $28(70.0)$ & 0,62 & $0,83(0,40-1,73)$ \\
\hline Não & $80(33,9)$ & $156(66,1)$ & & \\
\hline \multicolumn{5}{|l|}{ Drogas } \\
\hline Sim & $1(25.0)$ & $3(75.0)$ & 0,72 & $0,66(0,06-6,46)$ \\
\hline Não & $91(33,5)$ & $181(66,5)$ & & \\
\hline \multicolumn{5}{|l|}{ Drogas na gestação } \\
\hline Sim & $4(21,1)$ & $15(78,9)$ & 0,17 & $0,51(0,16-1,58)$ \\
\hline Não & $88(34,2)$ & $169(65,8)$ & & \\
\hline
\end{tabular}

${ }^{*} \mathrm{X}^{2}$

foi mais prevalente e $80,1 \%$ dos partos foram a termo. Quanto à história gestacional, 73,9\% das mulheres já haviam engravidado uma vez, 33,7\% tiveram parto pregresso e $88 \%$ não tinham história de abortamento. A maioria das puérperas não teve complicações relacionadas diretamente ao trabalho de parto ou à cesariana (96,7\%). Os dados referentes à história clínica e obstétrica estão presentes na Tabela 3.

Dentre as morbidades maternas graves consideradas casos, a análise dos distúrbios hipertensivos mostrou os seguintes percentuais: $10,1 \%$ tiveram hipertensão grave, seguido de $9,4 \%$ com pré-eclâmpsia grave, $0,4 \%$ com pré-eclâmpsia grave e hipertensão grave, e $0,4 \%$ com eclâmpsia. Dentre os distúrbios hemorrágicos, o descolamento prematuro de placenta teve uma maior prevalência, com $8,3 \%$, seguido pela hemorragia pós-parto com $0,7 \%$. Placenta acreta (considerada uma complicação relacionada ao momento do parto propriamente dito), prenhez ectópica e rotura uterina somaram $1,1 \%$. Apenas $2,2 \%$ das mulheres apresentaram insuficiência respiratória. Já os casos considerados near miss somaram um percentual de $2,3 \%$, subdivididos em: 0,4\% eclâmpsia e síndrome HELLP; 0;4\% parada cardíaca; $0,4 \%$ tireotoxicose; $0,4 \%$ embolia pulmonar; $0,4 \%$ complicações do SNC relacionadas à anestesia durante o parto; $0,7 \%$ crise convulsiva. Nenhuma das puérperas apresentou disfunção renal, hepática, hematológica ou hemostática. Foi verificado, no estudo, um óbito $(0,4 \%)$ por tromboembolismo pulmonar, complicação de pré-eclâmpsia grave. Não foram verificados óbitos entre os controles.

$\mathrm{Na}$ análise multivariada do modelo final de regressão logística, permaneceram as variáveis "história pregressa de hipertensão arterial", "prematuridade" e "parto cesáreo". Essas variáveis se 
Tabela 3. Distribuição de casos e controles de morbimortalidade materna de acordo com a história clínica e obstétrica, em Barbacena, Minas Gerais, 2014

\begin{tabular}{|c|c|c|c|c|}
\hline Variáveis & Casos & Controles & Valor $\mathbf{p}^{*}$ & OR (95\%IC) \\
\hline & n (\%) & n (\%) & & \\
\hline \multicolumn{5}{|c|}{ Uso de anti-hipertensivos } \\
\hline Sim & $37(88,1)$ & $5(11,9)$ & 0,00 & $24,08(9,02-64,26)$ \\
\hline Não & $55(23,5)$ & $179(76,5)$ & & \\
\hline \multicolumn{5}{|l|}{ Vacinação completa } \\
\hline Não & $3(33,3)$ & $6(66,7)$ & 1,00 & $1,0(0,24-4,09)$ \\
\hline Sim & $89(33,3)$ & $178(66,7)$ & & \\
\hline \multicolumn{5}{|l|}{ Diabetes prévio } \\
\hline Sim & $4(80,0)$ & $1(20,0)$ & 0,04 & $8,25(1,02-206,8)$ \\
\hline Não & $88(32,5)$ & $183(67,5)$ & & \\
\hline \multicolumn{5}{|l|}{ Hipertensão prévia } \\
\hline Sim & $20(83.3)$ & $4(16.7)$ & 0,00 & $12,5(4,12-37,8)$ \\
\hline Não & $72(28,6)$ & $180(71,4)$ & & \\
\hline \multicolumn{5}{|l|}{ Dislipidemia prévia } \\
\hline Sim & $2(66,7)$ & $1(33.3)$ & 0,25 & $4,06(0,36-45,4)$ \\
\hline Não & $90(33,0)$ & $183(67,0)$ & & \\
\hline \multicolumn{5}{|l|}{ Gestações pregressas } \\
\hline Nenhuma & $24(35,3)$ & $44(64,7)$ & 0,03 & $1,58(0,83-3,01)$ \\
\hline Uma & $37(42,5)$ & $50(50,7)$ & & $2,14(1,19-3,87)$ \\
\hline$>$ Duas & $31(25,6)$ & $90(74,4)$ & & - \\
\hline \multicolumn{5}{|l|}{ Partos pregressos } \\
\hline Não & $26(36,1)$ & $46(63,9)$ & 0,02 & $1,75(0,92-3,36)$ \\
\hline Um & $39(41,9)$ & $54(58,1)$ & & $2,24(1,23-4,08)$ \\
\hline$\geq 2$ & $27(24,3)$ & $84(75,7)$ & & - \\
\hline \multicolumn{5}{|l|}{ Abortos prévios } \\
\hline Sim & $14(42,4)$ & $19(57,6)$ & 0,23 & $1,55(0,74-3,27)$ \\
\hline Não & $78(32,1)$ & $165(67,9)$ & & \\
\hline \multicolumn{5}{|l|}{ Número de consultas } \\
\hline $0-3$ & $6(50,0)$ & $6(50,0)$ & 0,44 & $1,93(0,53-7,03)$ \\
\hline $4-5$ & $15(34,1)$ & $29(65,9)$ & & $1,08(0,54-2,15)$ \\
\hline$\geq 6$ & $71(32,3)$ & $149(67,7)$ & & - \\
\hline \multicolumn{5}{|l|}{ Tipo de parto } \\
\hline Cesariana & $75(42,4)$ & $102(57,6)$ & 0,00 & $3,54(1,94-6,47)$ \\
\hline Vaginal & $17(17,2)$ & $82(82,8)$ & & \\
\hline \multicolumn{5}{|l|}{ A termo } \\
\hline Não & $39(70,9)$ & $16(29,1)$ & 0,00 & $7,72(3,99-14,93)$ \\
\hline Sim & $53(24,0)$ & $168(76,0)$ & & \\
\hline
\end{tabular}

${ }^{*} \mathrm{x}^{2}$

associaram com maior chance de desenvolvimento de "near miss" (Tabela 4). O modelo mostrou bom ajuste aos dados de acordo com o teste de Hosmer e Lemeshow $(p=0,871)$

\section{DISCUSSÃO}

$\mathrm{Na}$ análise bivariada, as variáveis que se mostraram associadas à ocorrência de agravos à saúde foram a história pregressa de hipertensão arterial, parto prematuro, tipo de parto, uso controlado de medicamentos anti-hipertensivos e diabetes mellitus prévio.

Parturientes com história pregressa de hipertensão arterial tiveram maior chance de desenvolver morbidades maternas graves, como pré-eclâmpsia, eclâmpsia e síndrome HELLP, que são complicações frequentes na gestação. A associação entre as síndromes hipertensivas e a história pregressa de hipertensão arterial é endossada pela literatura ${ }^{1,18,19}$. A hipertensão crônica é uma situação médica que acarreta alto risco gestacional, já que representa a principal causa de mortalidade materna. Por isso devem-se priorizar a prevenção e o tratamento desse agravo, requerendo maior vigilância durante a gravide $z^{18,20}$.

A prematuridade ocorre, principalmente, naquelas mulheres que apresentam descolamento prematuro de placenta, hemorragia, pré-eclâmpsia, eclâmpsia, hipertensão grave e diabetes mellitus gestacional. Essas condições podem comprometer a saúde 
Tabela 4. Fatores de risco para morbimortalidade materna na análise multivariada, em Barbacena, Minas Gerais, 2014

\begin{tabular}{|c|c|c|c|c|}
\hline Variáveis & Casos & Controles & Valor p & OR (95\%IC) \\
\hline & n (\%) & n (\%) & & \\
\hline \multicolumn{5}{|l|}{ Hipertensão prévia } \\
\hline Sim & $20(83.3)$ & $4(16.7)$ & 0,00 & $14,3(4,5-45,5)$ \\
\hline Não & $72(28,6)$ & $180(71,4)$ & & \\
\hline \multicolumn{5}{|l|}{ Tipo de parto } \\
\hline Cesariana & $75(42,4)$ & $102(57,6)$ & 0,00 & $3,2(1,6-6,3)$ \\
\hline Vaginal & $17(17,2)$ & $82(82,8)$ & & \\
\hline \multicolumn{5}{|l|}{ A termo } \\
\hline Não & $39(70,9)$ & $16(29,1)$ & 0,00 & $7,8(3,9-15,8)$ \\
\hline Sim & $53(24,0)$ & $168(76,0)$ & & \\
\hline
\end{tabular}

materna e fetal, havendo a necessidade da interrupção precoce da gestação. Essa relação é corroborada por outros estudos ${ }^{19,20,21}$. Além disso, a elevada frequência de partos prematuros no presente estudo pode ser explicada pelo fato de o hospital ser o único da microrregião com unidade de terapia intensiva neonatal.

No Sistema Único de Saúde (SUS), a escolha da mulher sobre o tipo de parto é limitada, diferentemente no setor privado, no qual a mulher tem a possibilidade de agendamento de uma cesariana, conforme desejo particular e/ou indicação obstétrica. No SUS, as gestantes, geralmente, são acompanhadas por diferentes profissionais na assistência ao pré-natal, e, na maioria das vezes, a cesariana é realizada mediante o diagnóstico de intercorrências durante a gestação ou trabalho de parto, sem muita possibilidade de agendamento por vontade da própria mulher ${ }^{22}$. Em relação ao número de cesarianas, foi verificada associação entre o procedimento e a ocorrência de morbidades maternas, como o tromboembolismo. A maior ocorrência de infecção e de hemorragia, que são duas das principais causas de agravos às condições maternas, não foi vista no presente estudo, ao contrário do que é encontrado na literatura ${ }^{23,24}$. A necessidade de interrupção imediata das gestações ocorre devido à presença de doenças obstétricas de maior gravidade nessas pacientes, nas quais apenas a resolução da gravidez irá evitar maiores complicações. Como nem sempre é possível ou seguro induzir o trabalho de parto vaginal em tais casos, a realização de cesariana é requerida, o que também explica o índice encontrado. Apesar de o parto cesáreo estar associado à morbimortalidade materna, quando comparado ao parto vaginal, a interrupção das gestações, quando bem indicada, pode contribuir para redução do risco de óbito do binômio materno-fetal ${ }^{20,21,25}$.

Quanto à história de parto pregresso, houve uma associação entre uma gestação prévia e o desenvolvimento de near miss, quando comparado às mulheres sem histórico de gestações anteriores ou com mais de duas gestações prévias. O maior risco de morbidades maternas em multíparas pode ser confirmado por outros estudos ${ }^{26-29}$. Essa relação é reforçada pelo fato de que morbidades em gestações anteriores tendem a se repetir nas próximas gestações. Como exemplo dessa tendência, podemos citar o diabetes gestacional e a doença hipertensiva específica da gravidez, que são uma das causas mais comumente associadas à morbidade materna grave e o near miss ${ }^{20}$. Porém não foram localizados estudos relacionando o desenvolvimento de complicações com o histórico de apenas uma gestação pregressa. Outro dado importante é que a idade materna avançada, em geral, tanto em primíparas quanto em multíparas, relaciona-se com o desenvolvimento de morbimortalidade ${ }^{19,23,30}$, relação não observada na amostra pesquisada.

O uso controlado de medicamentos anti-hipertensivos durante a gestação também se mostrou associado ao desfecho pesquisado neste estudo. Isso poderia ser explicado pelo fato de que nem todos os grupos dessas drogas são isentos de risco na gestação, trazendo complicações tanto para a mãe quanto para o feto. Apesar de o objetivo do uso desses medicamentos ser o de controlar a doença presente, esse fator representa um possível causador de morbimortalidade iatrogênica. Além disso, a gestante em uso dessas drogas objetiva controlar a doença hipertensiva, que por si só mostrou relação com o desenvolvimento de complicações ${ }^{24}$. Por isso deve-se também considerar a possibilidade de que as pacientes em uso desses medicamentos não estavam com a doença hipertensiva controlada, por exemplo, devido à inadequação terapêutica a essa gestante ou pelo seu uso irregular.

No presente estudo, observou-se associação entre diabetes e morbidade materna, apesar do diabetes mellitus não fazer parte dos critérios adotados pela OMS. Sabe-se que essa variável implica repercussões importantes materno-fetais, haja vista que o mau controle metabólico resulta em maiores índices de infecções, hipertensão arterial, pré-eclâmpsia, pré-termo e de cesarianas em casos de macrossomia fetal. É uma doença relacionada à hipertensão arterial, que foi uma das variáveis significativas no presente estudo, já que possuem fatores de risco em comum; já o diabetes relaciona-se a danos renais potenciais causadores de elevação da pressão arterial, além de danos vasculares ${ }^{31-33}$.

Neste estudo, algumas variáveis não se relacionaram ao maior risco de desenvolvimento de morbimortalidade materna, apesar 
de serem citadas na literatura sobre o tema. Antecedente de aborto, idade materna avançada e ter realizado menos do que seis consultas de pré-natal são considerados fatores de risco para o near miss materno, de acordo com alguns estudos ${ }^{1,20,23,25,34}$. Além desses fatores, o baixo nível socioeconômico também não foi um fator relacionado ao maior risco materno no presente estudo, já que a maioria das pacientes se enquadrava em um mesmo nível de baixa renda ${ }^{9}$. Também não foi possível comparar a incidência de near miss materno em gestantes atendidas por diferentes serviços, pois foram incluídas no estudo apenas aquelas atendidas pelo SUS no mesmo hospital.

O presente estudo evidenciou algumas limitações que devem ser consideradas. A principal se refere ao viés de memória, o que é uma ocorrência esperada em estudos caso-controle. Além disso, o questionário foi conduzido por diferentes entrevistadores, o que implica em diferenças na condução das entrevistas e na interpretação das perguntas e resultados observados. Esses dados foram minimizados pelo treinamento prévio dos pesquisadores. Outros fatores que podem ter sido limitantes foram características momentâneas das entrevistadas (estado de fadiga e humor) e possíveis omissões ou respostas inverídicas pelo próprio estado de saúde ou pela presença de terceiros.

\section{CONCLUSÃO}

Confirmou-se neste estudo para nível local o que é apresentado na literatura para o quadro nacional. Os resultados deste estudo apontaram algumas variáveis clínicas e obstétricas associadas à presença de eventos adversos nas puérperas, ressaltando que, em sua maioria, são fatores passíveis de prevenção ou de tratamento.

Essa casuística reforça a importância da realização de pré-natal de no mínimo seis consultas, assim como é preconizado pela OMS, para que haja a identificação precoce das gestantes de alto risco, a fim de encaminhá-las para os centros de referência obstétrica. Porém, mais do que a quantidade de consultas, o presente estudo mostrou uma importância ainda maior da qualidade da atenção pré-natal. As equipes de saúde devem estar efetivamente preparadas para reconhecer os fatores de risco que levam à morbimortalidade materna. Desse modo, poderá haver uma melhora da qualidade do atendimento obstétrico, contribuindo para redução do near miss na microrregião estudada.

\section{REFERÊNCIAS}

1. Morais APP, Barreto SM, Passos VMA, Golino PS, Costa JE, Vasconcelos MX. Severe maternal morbidity: a case-control study in Maranhão, Brazil. Reprod Health. 2013;10(1):11. http://dx.doi.org/10.1186/1742-4755-10-11. PMid:23399443.

2. World Health Organization - WHO. Trends in maternal mortality: 19902008. Geneva; 2010.

3. Viana RC, Novaes MRCG, Calderon IMP. Mortalidade materna: uma abordagem atualizada. Cienc Saúde. 2011;22:141-52.

4. Souza JP, Cecatti JG, Parpinelli MA, Sousa MH, Serruya SJ. Revisão sistemática sobre morbidade materna near miss. Cad Saude Publica. 2006;22(2):255-64. http://dx.doi.org/10.1590/S0102-311X2006000200003. PMid:16501738.

5. World Health Statistics - WHO. World Health Statistics 2012 [Internet]. Geneva; 2012 [citado em 2013 out 3]. Disponível em: http://www.who.int/ gho/publications/world_health_statistics/2012/en/

6. Nakamura-Pereira M, Mendes-Silva W, Dias MAB, Reichenheim ME, Lobato G. Sistema de informações hospitalares do Sistema Único de Saúde (SIH-SUS): uma avaliação do seu desempenho para a identificação do near miss materno. Cad Saude Publica. 2013;29(7):1333-45. http://dx.doi. org/10.1590/S0102-311X2013001100008. PMid:23843001.

7. Szwarcwald CL, Escalante JJC, Rabello No DL, Souza Jr PRB, Victora CG. Estimação da razão de mortalidade materna no Brasil, 2008-2011. Cad Saude Publica. 2014;30:S71-83. http://dx.doi.org/10.1590/0102-311X00125313.

8. Vidal CEL, Carvalho MB, Machado MGS, Coelho MTC, Carvalho LRO. Mortalidade de mulheres em idade fértil na microrregião de Barbacena. In: Anais do Fórum Científico da Fhemig; 2014; Belo Horizonte. Belo Horizonte: Fhemig; 2014.

9. Souza JP, Cecatti JG, Haddad SM, Parpinelli MA, Costa ML, Katz L, et al. The WHO maternal near-miss approach and the maternal severity index model (MSI): tools for assessing the management of severe maternal morbidity. PLoS One. 2012;7(8):44-129. http://dx.doi.org/10.1371/journal. pone.0044129. PMid:22952897.

10. Tunçalp O, Hindin MJ, Souza JP, Chou D, Say L. The prevalence of maternal near miss: a systematic review. BJOG. 2012;119(6):653-61. http://dx.doi. org/10.1111/j.1471-0528.2012.03294.x. PMid:22489760.

11. Brasil. Ministério da Saúde. Estudo da mortalidade de mulheres de 10 a 49 anos, com ênfase na mortalidade materna. Brasília; 2006.

12. Souza JPD, Cecatti JG, Parpinelli MA. Fatores associados à gravidade da morbidade materna na caracterização do near miss. Rev Bras Ginecol Obstet. 2005;27(4):197-203. http://dx.doi.org/10.1590/S0100-72032005000400006.

13. Souza JP, Gulmezoglu AM, Carroli G, Lumbiganon P, Qureshi Z. The World Health Organization multicountry survey on maternal and new born health: study protocol. BMC Health Serv Res. 2011;11(1):286. http:// dx.doi.org/10.1186/1472-6963-11-286. PMid:22029735.

14. Lotufo FA, Parpinelli MA, Haddad SM, Surita FG, Cecatti JG. Applying the new concept of maternal near-miss in an intensive care unit. Clinics. 2012;67(3):225-30. http://dx.doi.org/10.6061/clinics/2012(03)04. PMid:22473402.

15. Brasil. Ministério da Saúde. Indicadores de cobertura [Internet]. Brasília; 2011 [citado em 2014 out 30]. Disponível em: http://tabnet.datasus.gov. br/cgi/tabcgi.exe?idb2012/f06.def

16. Lasky T, Stolley PD. Selection of cases and controls: applications of the case-control method. Epidemiol Rev. 1994;16(1):13. PMid:7925728.

17. Luiz RR. Associação estatística em epidemiologia: análise com múltiplas variáveis. In: Medronho RA, editor. Epidemiologia. São Paulo: Atheneu; 2009. p. 457-483.

18. Luz AG, Tiago DB, Silva JCG, Amaral E. Morbidade materna grave em um hospital universitário de referência municipal em Campinas, Estado 
de São Paulo. Rev Bras Ginecol Obstet. 2008;30(6):281-6. http://dx.doi. org/10.1590/S0100-72032008000600003. PMid:19142505.

19. Pacheco AJC, Katz L, Souza ASR, Amorim MMR. Factors associated with severe maternal morbidity and near miss in the São Francisco Valley, Brazil: a retrospective, cohort study. BMC Pregnancy Childbirth. 2014;14(1):91. http://dx.doi.org/10.1186/1471-2393-14-91. PMid:24576223.

20. Morse ML, Fonseca SD, Gottgtroy CL, Waldmann CS, Gueller E. Morbidade materna grave e Near Miss em hospital de referência regional. Rev Bras Epidemiol. 2011;14(2):310-22. http://dx.doi.org/10.1590/S1415790X2011000200012. PMid:21655697.

21. Oliveira LC, Costa AAR. Óbitos fetais e neonatais entre casos de near miss materno. Rev Assoc Med Bras. 2013;59(5):487-94. http://dx.doi. org/10.1016/j.ramb.2013.08.004. PMid:24080346.

22. Domingues RMSM, Dias MAB, Nakamura-Pereira M, Torres JA, d'Orsi E, Pereira APE, et al. Processo de decisão pelo tipo de parto no Brasil: da preferência inicial das mulheres à via de parto final. Cad Saude Publica. 2014;30(Supl 1):S101-16. http://dx.doi.org/10.1590/0102-311X00105113.

23. Oliveira FC Jr, Costa ML, Cecatti JG, Pinto e Silva JL, Surita FG. Maternal morbidity and near miss associated with maternal age: the innovative approach of the 2006 Brazilian demographic health survey. Clinics. 2013;68(7):922-7. http://dx.doi.org/10.6061/clinics/2013(07)06. PMid:23917654.

24. Osorio-de-Castro CGS, Paumgartten FJR, Silver LD. O uso de medicamentos na gravidez. Cien Saude Colet. 2004;9(4):987-96. http://dx.doi.org/10.1590/ S1413-81232004000400019.

25. Amorim MM, Katz L, Valença M, Araújo DE. Morbidade materna grave em UTI obstétrica no Recife, região nordeste do Brasil. Rev Assoc Med Bras. 2008;54(3):261-6. http://dx.doi.org/10.1590/S0104-42302008000300021. PMid:18604406.

26. Wianwiset W. Maternal near miss (severe morbidity) at Sisaket Hospital. Thai Journal of Obstetrics and Gynaecology. 2012;20(2):69-76.
27. Simonsen SE, Varner MW. Grand multiparity. United States: UpToDate; 2014 [citado em 2014 set 30]. Disponível em: http://www.uptodate.com/ contents/grand-multiparity? source $=$ machineLearning\&search $=$ grand $+\mathrm{m}$ ultiparity+multuparity\&selectedTitle $=1 \sim 12 \&$ sectionRank $=1$ \&anchor $=\mathrm{H}$ 2\#H8

28. Alsammani MA, Ahmed SR. Grandmultiparity: risk factors and outcome in a tertiary hospital: a comparative study. Med Arch. 2015;69(1):38-41. http://dx.doi.org/10.5455/medarh.2015.69.38-41. PMid:25870476.

29. Mgaya AH, Massawe SN, Kidanto HL, Mgaya HN. Grand multiparity: is it still a risk in pregnancy? BMC Pregnancy Childbirth. 2013;13(1):241. http://dx.doi.org/10.1186/1471-2393-13-241. PMid:24365087.

30. Gravena AAF, Paula MG, Marcon SS, Carvalho MDB, Pelloso SM. Idade materna e fatores associados a resultados perinatais. Acta Paul Enferm. 2013;26(2):130-5. http://dx.doi.org/10.1590/S0103-21002013000200005.

31. Montenegro Jr RM, Paccola GMFG, Faria CM, Sales APM, Montenegro APDR, Jorge SM, et al. Evolução materno-fetal de gestantes diabéticas seguidas no HC-FMRP-USP no Período de 1992-1999. Arq Bras Endocrinol Metabol. 2001;45(5):467-74. http://dx.doi.org/10.1590/S0004-27302001000500010.

32. Godoy SR, Gualda DMR, Bergamasco RB, Tsunechiro MA. Percepção da causalidade e dos sinais de near miss atribuídos por mulheres sobreviventes. Texto Contexto Enferm. 2008;17(3):536-43. http://dx.doi.org/10.1590/ S0104-07072008000300015.

33. Avenant T. Neonatal near miss: a measure of the quality of obstetric care. Best Pract Res Clin Obstet Gynaecol. 2009;23(3):369-74. http://dx.doi. org/10.1016/j.bpobgyn.2008.12.005. PMid:19201262.

34. Dias MAB, Domingues RMSM, Schilithz AOC, Nakamura-Pereira M, Diniz CSG, Brum IR, et al. Incidência do near miss materno no parto e pós-parto hospitalar: dados da pesquisa Nascer no Brasil. Cad Saude Publica. 2014;30(1):169-81. http://dx.doi.org/10.1590/0102-311X00154213.

Recebido em: Set. 23, 2015 Aprovado em: Jun. 14, 2016 